\title{
Emprendimiento e innovación en Ecuador, análisis de ecosistemas empresariales para la consolidación de pequeñas y medianas empresas
}

\section{Entrepreneurship and innovation in Ecuador, analysis of business ecosystems for the consolidation of small and medium enterprises}

Camino Guaján Rafael Charly

Universidad Central del Ecuador, Ecuador

Aguilar Viteri Andrés Esteban

Universidad Central del Ecuador, Ecuador

Autor para correspondencia: rcamino@uce.edu.ec, aeaguilar@uce.edu.ec Fecha de recepción: 01 de Junio de 2017 - Fecha de aceptación: 15 de Agosto de 2017

Resumen: El artículo presenta la continuación de la propuesta presentada en el CIICAE-UIDE 2017, Loja-Ecuador, 17 al 19 de mayo 2017, a través de un análisis descriptivo del emprendimiento desde una perspectiva global sectorial y local; parte de su epistemología hasta su impacto en la economía, las variantes de empresas que se impulsan fruto del emprendimiento e incorporación de la innovación, así como el análisis del impacto de la política pública relacionado con los resultados del Global Entrepreneurship Monitor de los años 2014, 2015 y 2016. La determinación del escenario actual a través del análisis de ecosistemas empresariales para el caso ecuatoriano define el punto de partida para la consolidación de proyectos empresariales nacientes, negocios nuevos y aporte fundamental para el desarrolla de la pequeña y mediana empresa.

Palabras clave: emprendimiento - ecuador; PYMES; empresa; factores para el emprendimiento; estructuras organizacionales

Abstract: The article presents the continuation of the proposal presented at CIICAE-UIDE 2017, Loja-Ecuador, from May 17 to 19, 2017, through a descriptive analysis of entrepreneurship from a global sectoral and local perspective; Part of its epistemology to its impact on the economy, the variants of companies that are driven by entrepreneurship and incorporation of innovation, as well as the analysis of the impact of public policy related to the results of the Global Entrepreneurship Monitor of 2014, 2015 and 2016. The determination of the current scenario through the analysis of business ecosystems for the Ecuadorian case defines the starting point for the consolidation of nascent business projects, new businesses and a fundamental contribution for the development of small and medium enterprises.

Key words: entrepreneurship - ecuador; SMEs; enterprise management model; organizational structures; factors for entrepreneurship 


\section{Introducción}

\section{Problema}

El emprendimiento como eje del desarrollo económico contempla la actividad que en la última década ha delimitado ciertos comportamientos dentro del esquema social ecuatoriano; determinar lineamientos para la gestión de las PYME'S y en especial su permanencia en el tiempo contempla el problema principal que afecta la gestión empresarial ecuatoriana, la identificación de factores claves para el desarrollo local de nuevos negocios fundamenta la importancia y alto impacto del estudio.

\section{Estado del Arte}

\section{Enunciaciones}

Partiendo de la conceptualización de emprendimiento desde su etimología el término emprendedor proviene de la palabra castellana emprender, del latín Apprehendere; Determinarse a tratar y hacer alguna cosa ardua y dificultosa: como una facción militar, una conquista, etc. (REAL ACADEMIA ESPAÑOLA, 1732)

La evolución del vocablo se relaciona con el término francés entrepreneur a inicios del siglo XVI, contemplando en el siglo XVIII una extensión al significado del término a los constructores y arquitectos. En 1803 Say en su tratado de Economía Política define entrepreneur a la persona que dirige una empresa, especialmente un contratista actuando como intermediario entre capital y trabajo.

Joseph Schumpeter en 1911 sugiere que la innovación e invención son la clave del crecimiento económico determinado por los emprendedores, desarrollando una teoría de investigación referente a la economía y empresa con la corriente económica, socio psicológica y comportamental.

Según la corriente económica podemos ver al emprendimiento como un proceso de combinaciones de productos, servicios y procesos que se convierten en un referente de cambio económico a través del aprovechamiento de oportunidades y la innovación. (Drucker, 1985)

A partir del corriente socio psicológico se define al emprendedor desde una perspectiva personal, con características, competencias y rasgos intrínsecos como la creatividad, la capacidad de asumir riesgos, independencia, interdependencia y necesidad al logro de objetivos. (McClelland, 1961)

En estudios más recientes a partir de 1999 el Global Entrepreneurship Monitor (GEM) examina al emprendimiento desde la generación de una idea, las acciones para su ejecución, evaluación, consolidación en el mismo y valoración cuando sobrevive más de tres años y medio en el mercado.

Así también se contempla el emprendimiento corporativo como actividades innovadoras que permiten generar y sostener ventajas competitivas con el uso óptimo de recursos adaptados a 
la fluctuación de escenarios, no como la simple creación de empresas sino ampliando su espectro a la orientación de estrategias empresariales en base al crecimiento e innovación, identificación de oportunidades económicas, desarrollo de nuevos productos, nuevos diseños organizacionales, nuevos procesos de producción, adaptación o desarrollo de canales de distribución orientados a las nuevas iniciativas en los mercados manteniendo la premisa de optimización de recursos, generación de competitividad y amplia rentabilidad.

\section{El emprendimiento en la economía}

A partir de 2003 Michelacci propone un modelo de crecimiento endógeno donde la figura del emprendedor así como el cambio tecnológico son el pilar fundamental para la generación de nuevas ideas; también demuestra que el emprendedor asigna recursos a su proyecto en función al rendimiento de la innovación; estableciendo al emprendimiento como un mecanismo para convertir el conocimiento en un factor económicamente rentable; siendo un determinante relevante para el desarrollo económico a través de la transformación de retos en oportunidades, generación de trabajo, competitividad, productividad, cambio tecnológico e innovación.

En este sentido se define a la innovación como el motor de desarrollo socioeconómico y al emprendimiento como la herramienta para la consolidación de estos procesos; en este esquema se determina los Sistemas de Innovación (SI) como los espacios donde se producen procesos de emprendimiento, gestión, aprendizaje y generación de conocimiento económicamente rentable.

Pensando en el crecimiento económico según lo señalan Gong y Killer en el 2003, para el caso de un país rico el crecimiento se encuentra en función de la producción vinculado al desarrollo tecnológico, mientras que para el caso de países que poseen gran cantidad de recursos no explotados el crecimiento se encuentra en función a la movilización de los mismos generando especialización en imitación tecnológica desarrollada en cualquier otro país.

\section{Definición y tipos de empresas en el entorno del emprendimiento}

Tras analizar parte del entorno económico en función del emprendimiento es necesario considerar los tipos de empresa que se consolidan en este esquema, adaptando definiciones para una correcta pauta en el desarrollo de nuevos proyectos.

Empresa de Base Tecnológica (EBT); constituyen los negocios propios e independientes centrados en la explotación de un invento o innovación tecnológica, contemplándose así pequeñas empresas que operan en áreas tecnológicas emergentes de amplio cambio innovador a través del uso intensivo de conocimiento científico y técnico. (Simón, 2003)

Start Up; constituyen las empresas que están en su etapa de introducción en el mercado, creativas con diferenciación enmarcadas en la innovación y desarrollo tecnológico. Este tipo de empresas constituyen un apoyo económico para la creación de fuentes de trabajo especializadas, enmarcan su gestión en dar solución a procesos complicados a través de la innovación. Esta nomenclatura de empresa se da únicamente a este tipo de empresas en su fase de introducción, cuando estas se consolidan y llegan a la fase de crecimiento y madurez dejan de ser consideradas Start Up. (Shane, 2002) 
Spin Off; Se define a este tipo de empresa como la conformación de una empresa a partir de la oportunidad percibida por su empresa matriz, su conformación nace de un equipo de trabajo a partir de la empresa base; enfocándose ampliamente en explotar las oportunidades conseguidas a partir de los resultados desarrollados u obtenidos. Una subclase de las empresas Spin Off son las desarrolladas a partir de la asesoría universitaria brindada por profesores investigadores, personal técnico y de servicios, así como las promovidas por los estudiantes como resultados de la ejecución de sus proyectos finales de carrera, denominándose en este sentido a este tipo de empresas Spin Off Universitaria definidas como el tipo de empresas creadas para explotar parte de la propiedad intelectual generada en una institución de educación superior. (Shane, 2002)

\section{Perspectivas de las Instituciones de Educación Superior en el marco del emprendimiento e innovación}

A partir de los procesos de evaluación implementados en Ecuador por el CEAACES desde el 2013 en las instituciones de educación superior (IES), se determina como premisa fundamental el mejoramiento de la calidad de la educación superior, identificando componentes primordiales que se involucran en la perspectiva actual y futura para el desarrollo científico, humanístico y tecnológico vinculado al emprendimiento.

Actualmente según el modelo genérico de evaluación del entorno del aprendizaje de carreras presenciales y semipresenciales de las universidades y escuelas politécnicas; se puede denotar con claridad el desarrollo de diversos criterios que involucran la innovación en el marco del emprendimiento donde las IES deben demostrar a partir de sus evidencias y planificaciones prospectivas su accionar en función de las necesidades de desarrollo local, regional y nacional; orientándose a las tendencias demográficas locales, provinciales, regionales; a la vinculación con una estructura productiva potencial de la provincia y región, y políticas nacionales de ciencia y tecnología. (LOES, 2010).

A partir del criterio de pertinencia se considera fielmente que el programa académico ofertado debe responder a las expectativas de la sociedad, planificación, prospectiva, desarrollo científico, humanístico y tecnológico. El mesocurrículo y microcurrículo determinan que las carreras y programas deben incluir en su planificación los campos de formación, redes, adaptaciones y vínculos transversales, que permitan abordar el aprendizaje de modo integrado e innovador. La calidad docente basa su atributo de acuerdo a la actualización científica y pedagógica, la innovación en procesos de enseñanza aprendizaje y la eficacia educativa; así también contempla de fundamental importancia la producción académica a partir del desarrollo del pensamiento universal, al despliegue de la producción científica y la promoción de la transferencia e innovación tecnológica.

Este enfoque de innovación contempla la gestión educativa universitaria y abre nuevas expectativas en los escenarios de la educación superior, definiendo oportunidades donde se asienten procesos de mejoramiento continuo, en un esquema de perfeccionamiento integrando a la investigación, emprendimiento y vinculación para el desarrollo de empresas Spin Offs Universitarias.

\section{Metodología}


El presente estudio muestra un análisis bibliográfico, exploratorio y descriptivo en base a datos del Global Entrepreneurship Monitor Ecuador - 2015 y Global Report 2016 - 2017; descripciones de estudios a nivel mundial, regional y local, perspectivas de alta relevancia que brindan un enfoque integral del Ecuador en el contexto mundial, acotejando la información a escenarios actuales del mismo.

En referencia al reporte GEM Ecuador 2015, los resultados obtenidos son desarrollados a través de la encuesta de población adulta (APS) que se aplica a 2,120 individuos cuyas edades están comprendidas entre los 18 y 93 años. La muestra se construye con representatividad nacional a partir de un muestreo estratificado autorepresentativo, toma en consideración el área de residencia (urbana o rural), el género y la edad de la población de referencia. Para la construcción del marco muestral se divide la población en 7 dominios construidos en base a la última información demográfica proyectada a partir del Censo de Población y Vivienda 2010. (GEM, 2015)

El Global Report 2016 - 2017 fundamenta sus resultados en base a 64 economías mundiales, a través de la encuesta de población adulta (APS) (entre los 18 y 64 años de edad) y 65 economías que desarrollaron la encuesta nacional de expertos (NES); los países encuestados en el 2016 representan el 69,2\% de la población mundial y el 84,9\% del PIB mundial. (GLOBAL ENTREPRENEURSHIP MONITOR, 2017)

\section{Resultados}

\section{El emprendimiento en un contexto mundial, regional y local}

Al analizar el emprendimiento desde una perspectiva mundial, The Global Entrepreneurship Monitor (GEM) consolida los informes de mayor relevancia que miden la iniciativa empresarial con información notable, alianzas en más de 100 países, 300 instituciones académicas y de investigación como Babson College, Universidad del Desarrollo, Universiti Tun Abdul Razak, Tecnológico de Monterrey y en Ecuador ESPAE - ESPOL, centrando los estudios en el análisis del comportamiento social, actitudes empresariales y contexto nacional.

En esta perspectiva según el informe 2016/17 GLOBAL REPORT más de dos tercios de los adultos en edad de trabajar consideran al emprendimiento como una buena elección de carrera donde perciben un desarrollo profesional, previendo alcanzar un estatus social alto como empresarios.

Centrando el análisis a nivel regional se puede describir al emprendimiento como una buena opción de carrera en América Latina y el Caribe tiene apenas un 17\% de aceptación entre los adultos en edad de trabajar; en Europa el 79\% de los países bajos lo consideran relevante; mientras que en Asia menos del 40\% lo determinan como buena opción de carrera; los contrastes señalados dan un reflejo de la realidad económica de las regiones en función a la percepción del emprendimiento y su mantenimiento en el tiempo, los mismos que se ven reflejados en función a sus economías delimitando tres segmentos marcados: por la explotación de materias primas, la eficiencia en la productividad y el desarrollo tecnológico e innovación. 
El emprendimiento es visto con un alto impacto en las economías de los factores productivos mismas que se caracterizan por una baja calificación laboral y explotación de recursos naturales, economías influenciadas por los precios dictados a nivel mundial y acontecimientos geopolíticos; una de las características más peculiares en estas economías es la creciente tasa de desempleo o subempleo siendo un factor primordial para el desarrollo de proyectos empresariales ligados más en la necesidad personal que en la oportunidad que brinda el entorno.

Por otro lado, se tiene a las economías de eficiencia impulsadas en los países donde la infraestructura y maquinaria son lo suficientemente fuertes con altos niveles de producción, fundamentan su accionar en altos estándares de productividad con una amplia optimización de recursos; y por último las economías impulsadas a través de la innovación, mismas que se desenvuelven en los países desarrollados donde la investigación y desarrollo tecnológico son la base para la transformación.

Referente al factor innovación, el emprendimiento tiene una perspectiva amplia y contrastante, el GEM evalúa los emprendimientos en función a la introducción de nuevos productos o servicios para clientes denotando la oferta de los mismos por pocos o ningún competidor; el aumento de los niveles de innovación en la mayoría de los casos se ve reflejado en función al nivel de desarrollo de los países. En este sentido los empresarios que se caracterizan en el desarrollo de este tipo de productos o servicios se enmarcan en entornos donde el acceso a tecnologías es acorde al mercado de soluciones tecnológicas avanzadas, con un alto nivel de educación en el factor humano.

A nivel regional se puede delimitar los niveles de innovación más altos en América del Norte y los más bajos en África; a nivel local resalta el caso de Chile e India reflejando en más de la mitad de los emprendimientos su caracterización en función a la innovación; así también las tasas más bajas de emprendimientos en innovación lo refleja Senegal y Bulgaria con un aporte de menos del $10 \%$.

Un contraste muy especial se refleja al evaluar la Tasa de Actividad Emprendedora (TEA), la cual mide los emprendimientos de entre 0 y 3,5 años en el mercado sobre la población de 18-64 años de un país, considerando este período como decisivo para la consolidación de los proyectos empresariales en el tiempo. Al evaluar la TEA con los niveles de innovación se refleja una relación inversamente proporcional denotando resultados en economías con altos niveles de TEA con bajos niveles de innovación, mientras que en economías con enfoque en la innovación según este reporte existen pocas personas que emprenden en nuevos proyectos empresariales, curiosamente los pocos que lo realizan tienen mayor propensión a exponer su oferta con un éxito mayor. Un caso especial que no cumple esta relación inversa es Chile y el Líbano donde se demuestra que los niveles de innovación van de la mano con la tasa de actividad emprendedora.

Otro factor relevante por evaluar es la internacionalización siendo considerada por los emprendedores a nivel mundial quienes manifiestan que del total de sus ventas más del 25\% provienen a través de fuentes fuera de su economía local; nuevamente las economías con enfoque en la innovación siendo las más desarrolladas resaltan el nivel promedio más alto de 
internacionalización con 20 puntos sobre 25 , le siguen las economías con enfoque en la eficiencia con 13 puntos y por último las economías de factores primarios contemplan apenas 6 puntos. Se puede manifestar que los empresarios de las economías desarrolladas tienen una previsión y olfato de negocio en función a las necesidades que se encuentran en el exterior, determinando mercados poco competitivos para sus productos o servicios; mientras que las economías subsiguientes delimitan su enfoque en función a las necesidades locales con poca rivalidad y opción a exportar, es decir resultan en función de la oportunidad local con enfoque internacional cuando se tiene la opción. A nivel regional se puede manifestar que Europa y América del Norte tienen los niveles más altos de internacionalización, mientras que los reportes más bajos se manifiestan en América Latina y el Caribe; a nivel general por cada región se puede estimar en promedio que una quinta parte de los empresarios reportan ventas fruto de la exportación de bienes o servicios, a nivel local Luxemburgo, Suiza, Croacia y Eslovenia presentan una tercera parte de ventas fruto de las exportaciones.

GEM identifica ciertos factores que determinan un ecosistema óptimo para el emprendimiento, denotando el acceso al financiamiento, políticas gubernamentales, impuestos y burocracia, programas de gobierno, nivel de educación y práctica empresarial, educación empresarial y práctica a nivel de postgrado, investigación y desarrollo (I+D), transferencia tecnológica, acceso a infraestructura comercial profesional, dinámicas de mercado internas, mercado interno, gravámenes, acceso a infraestructura física y de servicios, normas sociales y culturales.

En este contexto de manera global en una escala de 1 a 9 la infraestructura física recibió valores por encima de 6 , mientras que con valores menores a 4 se puntúan los factores educación empresarial, mercado interno, gravámenes, transferencia tecnológica e investigación y desarrollo. Las economías desarrolladas develan tener de manera más consolidada el ecosistema para el emprendimiento, mientras que las economías en función de la eficiencia luchan en condiciones menos favorables, sorprende en este aspecto la valoración de las economías en función de los factores productivos quienes se mantienen de manera general sobre las economías en función de la eficiencia; la educación empresarial y práctica a nivel de postgrado puntúa en 4.7 en estas y 4.5 en las economías en función de la innovación y eficiencia, en programas de emprendimiento gubernamentales se puntúa en 3,9 en la economías por factores productivos, 4.1 en economías por eficiencia y 4.7 en economías por innovación. A nivel individual destaca Suiza donde 11 de las 12 condiciones óptimas de emprendimiento puntúan entre los 10 valores más altos, Holanda mantiene 10 en alta puntuación, Malasia 8, Canadá y Luxemburgo 7. Resalta el factor normas sociales y culturales donde las diez economías mejor puntuadas se encuentran distribuidas en economías de innovación (Israel, EE.UU., Canadá, Suiza, Estonia), economías de eficiencia (Lebanon, Ecuador, Indonesia, Malasia) y economías de factores productivos (Filipinas). 


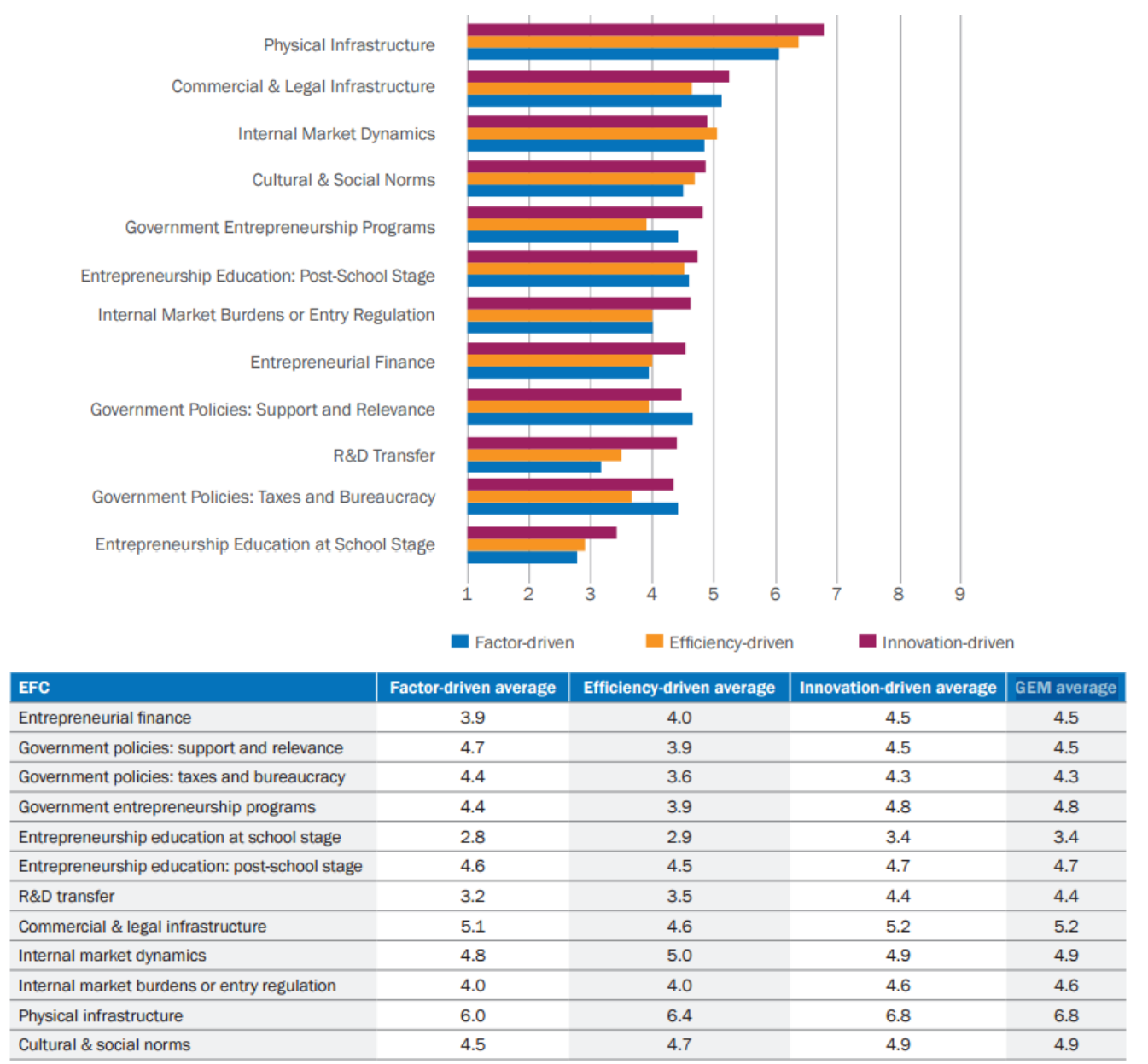

Figura 1: Promedios de la fase de desarrollo para los ecosistemas empresariales de 65 economías, GEM 2016 puntuaciones promedio

Fuente: (GLOBAL ENTREPRENEURSHIP MONITOR, 2017)

Referente a contextos a prospectivos en relación a EE.UU, la crisis financiera y la continua crisis global siguen siendo factores determinantes que se mantiene constantes en el escenario económico actual. El mundo se enfrenta a muchos retos, como el cambio climático, crecimiento del desempleo y una creciente dependencia de la tecnología en los negocios.

El desempleo y subempleo se han convertido en la principal preocupación de los países desarrollados y las economías en desarrollo. La población joven se constituye en una parte importante de la población en las economías en desarrollo, tomando en consideración la necesidad de los mismos por el alto índice de población en envejecimiento

Este informe indica los diversos perfiles de iniciativa empresarial en torno al mundo, los vacíos que se revelan y deben ser abordados con políticas y prácticas determinantes en cada una de las economías con enfoque prospectivo.

Los ecosistemas de emprendimiento difieren en gran medida entre los niveles de desarrollo de cada país. La disponibilidad de fondos y educación empresarial, el entorno normativo y el acceso a mercados se convierten en factores predominantes en cada economía. 
Estos resultados constituyen una valiosa herramienta de comparación entre países utilizándolos como referente y fuente de información (GLOBAL ENTREPRENEURSHIP MONITOR, 2017)

\section{El emprendimiento en Ecuador}

El análisis del emprendimiento en Ecuador marca una perspectiva de nuevos escenarios para el desarrollo de proyectos a largo plazo, el estudio de los resultados del último reporte Global Entrepreneurship Monitor Ecuador al 2015 delimita factores primordiales para una correcta comprensión y enfoque prospectivo.

Es preciso demarcar el escenario económico con el cual ha sido evaluado Ecuador, definiéndose como una economía basada en eficiencia, con una actividad emprendedora temprana mayor en toda la región (33.6\% frente al promedio de 19.9\%), y también como la más alta en relación a las economías en función de la eficiencia donde su media se encuentra en 15.2\%. Cabe mencionar que aunque este factor contempla una perspectiva altamente positiva según publicaciones de Leveraging Entrepreneurial Ambition and Innovation: A Global Perspective on Entrepreneurship, Competitiveness and Development, producida en conjunto por el World Economic Forum y el Global Entrepreneurship Monitor, y Bridging the Skills and Innovation Gap to boost Productivity in Latin America, del World Economic Forum Competitiveness Lab en colaboración con Deloitte se contempla que las economías que se encuentran en el primer cuartil con una TEA superior poseen baja competitividad, mientras que las economías que se encuentran en el último cuartil poseen alta competitividad; ajustándose ampliamente al escenario que vive Ecuador, más adelante se analizarán factores que demarcan esta premisa: TEA más alta de la región no corresponde proporcionalmente a un desarrollo económico,

Según este reporte el 35\% de los emprendedores se localizan en el área urbana, se observa un aumento en los emprendedores del área rural del $28 \%$ al 30\%, contemplando un $29.5 \%$ a emprendedores nacientes y un $9.9 \%$ a negocios nuevos; cabe mencionar la evolución de la TEA en los últimos años, denotando un incremento de un punto y una disminución del 17.7\% al $17.4 \%$ en la Tasa de Negocios Establecidos.

Figura 2: Evolución de la TEA en Ecuador

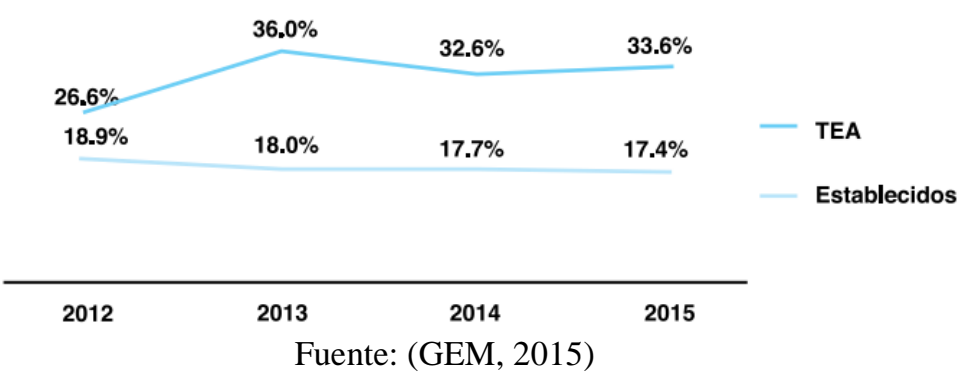

Un dato muy relevante para el análisis de la TEA, es que esta se clasifica por motivación en función a la oportunidad de mejora por la percepción de iniciar un negocio o coyuntura de mercado encontrada; y por necesidad la cual se manifiesta en función a la opción de no tener una mejor elección de trabajo; en este sentido la TEA por oportunidad corresponde al $68.8 \%$, 
mientras que la TEA por necesidad es del 30.6\%; este análisis define un valor por debajo de la media de economías de eficiencia la cual se mantiene en un $69.5 \%$; un tanto por encima del promedio de la región $68.2 \%$.

\begin{tabular}{|c|c|c|c|c|}
\hline & TEA & $\begin{array}{c}\text { TEA } \\
\text { Oportunidad }\end{array}$ & $\begin{array}{l}\text { TEA } \\
\text { Necesidad }\end{array}$ & $\begin{array}{c}\text { Índice } \\
\text { motivacional }\end{array}$ \\
\hline Ecuador & $33.6 \%$ & $68.8 \%$ & $30.6 \%$ & 1.1 \\
\hline Chile & $25.9 \%$ & $67.4 \%$ & $25.3 \%$ & 2.4 \\
\hline Colombia & $22.7 \%$ & $65.6 \%$ & $33.3 \%$ & 1.7 \\
\hline Perú & $22.2 \%$ & $72.9 \%$ & $25.2 \%$ & 2.1 \\
\hline Promedio Región & $19.9 \%$ & $68.2 \%$ & $29.6 \%$ & 1.9 \\
\hline $\begin{array}{l}\text { Promedio } \\
\text { Economías de Eficiencia }\end{array}$ & $15.2 \%$ & $69.5 \%$ & $28.3 \%$ & 2.0 \\
\hline
\end{tabular}

Figura 3: Comparación TEA Ecuador, Región / Economías de Eficiencia Fuente: (GEM, 2015)

Otro indicador fundamental en este contexto es el índice motivacional el cual se encuentra en 1.1, muy por debajo de la media de América Latina correspondiente a 1.9 y de la media de economías basadas en eficiencia con 2; esto refleja que en Ecuador se abren negocios por oportunidad y por necesidad casi a la par.

Estos datos reflejan con claridad que al año 2015 la economía ecuatoriana con precios de petróleo deficientes mantuvo indicadores de la TEA altos referente a la región y a las economías basadas en eficiencia, más esta no representa mayor importancia por cuanto los emprendimientos motivados por necesidad crecieron vorazmente lo cual no es óptimo, es claro delimitar que la falta de políticas, programas de gobierno, nivel de educación, práctica empresarial, (I+D), transferencia tecnológica y demás factores exógenos no fueron impulsados para la delimitación de oportunidades para consolidar escenarios con mayor fortaleza para el emprendimiento.

En base a los valores que la sociedad ecuatoriana refiere a una cultura pro emprendimiento según el reporte GEM Ecuador 2015 y el Global Report 2016-2017 se contempla una constante disminución del emprendimiento como una buena opción de carrera del $66.4 \%$ en el 2014, 61.6\% en el 2015, 59.5 \% en el 2016; la percepción del éxito en los negocios a través de un alto estatus también delimita una tendencia a la baja del 67,1\% en 2014 y 2015 hasta el $61.1 \%$ en el 2016; los valores mencionados se mantienen por debajo de la media de las economías basadas en eficiencia donde el $67 \%$ considera al emprendimiento como una buena opción de carrera y un alto estatus de éxito a los emprendedores exitosos.

Se determina de igual manera que para la continuidad de procesos y proyectos se debe articular esfuerzos aliados, de tal manera que se generen sinergias para apoyar a que los emprendedores e innovadores tengan acceso a:

1. Información de emprendimiento e innovación que permita a los emprendedores tomar decisiones acertadas y que mitiguen el riesgo, en lo relacionado a sus empresas.

2. Financiamiento de capital de riesgo que les permita a los emprendedores poner en marcha proyectos innovadores. 
3. Acceso a educación emprendedora para niños en escuelas y colegios; y desarrollo de competencias emprendedoras para profesionales. (Alianza para el Emprendimiento y la Innovación, 2014)

\section{Discusión}

Tras el análisis de los factores que determinan una visión sobre el mantenimiento de proyectos emprendedores en el tiempo con énfasis en los negocios nacientes y nuevos, el reporte (GLOBAL ENTREPRENEURSHIP MONITOR, 2017) delimita la base para la interpretación del escenario actual ecuatoriano en el entorno global, así como la determinación de propuestas para la toma de decisiones.

Como se había mencionado en la propuesta: "Emprendimiento, perspectiva global, regional y local; factores determinantes para la prospectiva de la gestión empresarial ecuatoriana" presentada en el Congreso Internacional de Investigación en Ciencias Administrativas y Económicas CIICAE UIDE 2017, los componentes que definen los factores críticos para la consolidación de los emprendimientos aún se encuentran con resultados por debajo de la región así como en relación a las economías de eficiencia donde se mantiene Ecuador en los últimos años.

Al analizar el indicador EEA (Actividad Emprendedora del Empleado) factor primordial que delimita la continuidad y permanencia en el tiempo de los nacientes y nuevos negocios, siendo un factor determinante para la generación de la gestión emprendedora dentro de la organización los resultados refieren cambios importantes: para el 2014 la EEA corresponde al 0.9\%, manteniéndose constante en el 2015, un cambio grave es el descenso de dos décimas en el 2016 donde la EEA se sitúa en el 0.7\%, al comparar el indicador con el promedio de la región para el $2016(2.3 \%)$ se percibe el alto declive siendo Ecuador y Perú los países con la EEA más baja de la región. Este aspecto es de alta preocupación para el emprendimiento en Ecuador denotando la inadecuada incorporación de personal sin procesos mínimos de selección, descuidando un factor primordial para la consolidación del negocio en el largo plazo.

Al revisar la TEA (Actividad Emprendedora Temprana), se registra un decremento en comparación al año 2015 bajando 1.7 puntos porcentuales; aun así, Ecuador se registra como el segundo país a nivel mundial con la TEA más alta. Cabe recalcar que el aspecto de mayor preocupación al analizar la TEA es el decremento de los negocios establecidos con de 3.2 puntos porcentuales es decir con un decremento del $22.53 \%$ en referencia al 2015. Esta comparación describe que a medida que se apertura nuevos negocios apenas el $44.65 \%$ de estos perduran en el tiempo.

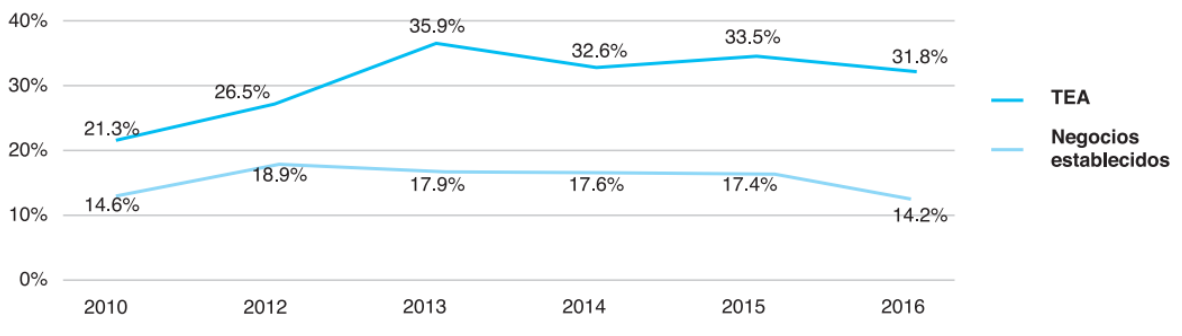


Figura 4: Tendencia TEA Ecuador - Negocios establecidos

Fuente: (GEM, 2017)

El tener una TEA elevada como ya se ha mencionado anteriormente no describe que el emprendimiento es efectivo, en base al análisis de los Global Report GEM 2014, 2015 y 2016 se ha determinado que la EEA es un factor concluyente para su consolidación en el tiempo, mientras se siga reclutando personal no calificado en nuevos emprendimientos estos perdurarán poco tiempo en el mercado.

Al analizar el ecosistema empresarial se delimitan factores relevantes para la consolidación de pequeñas y medianas empresas, el cuadro comparativo muestra la relación con economías altas, medias y bajas de la región, su promedio y relación con la media de economías de eficiencia.

\begin{tabular}{|c|c|c|c|c|c|c|c|}
\hline Factor & Descripción & Ecuador & Colombia & Chile & Perú & Región & $\begin{array}{l}\text { Economías de } \\
\text { Eficiencia }\end{array}$ \\
\hline A & Apoyo financiero & 2.86 & 3.63 & 3.53 & 3.83 & 3.42 & 4.07 \\
\hline B1 & Políticas gubernamentales: Políticas generales & 3.38 & 4.22 & 4.22 & 3.46 & 3.76 & 3.92 \\
\hline B2 & Políticas gubernamentales: Regulación & 2.68 & 3.60 & 4.74 & 3.14 & 3.31 & 3.67 \\
\hline $\mathrm{C}$ & Programas gubernamentales & 3.36 & 4.51 & 5.05 & 4.07 & 4.05 & 3.96 \\
\hline D1 & Educación y formación: Primaria y secundaria & 3.07 & 2.94 & 2.38 & 3.19 & 2.74 & 2.93 \\
\hline D2 & Educación y formación: Educación superior & 5.33 & 5.40 & 4.77 & 4.87 & 4.85 & 4.59 \\
\hline$E$ & Transferencia de I+D & 3.17 & 3.53 & 3.95 & 3.24 & 3.38 & 3.55 \\
\hline $\mathrm{F}$ & Infraestructura comercial y profesional & 4.57 & 4.16 & 4.92 & 3.65 & 4.50 & 4.72 \\
\hline G1 & Apertura del mercado interior: Dinámica & 4.54 & 4.65 & 3.73 & 4.25 & 4.39 & 5.08 \\
\hline G2 & Apertura del mercado interior: Apertura & 4.21 & 3.85 & 3.99 & 4.01 & 3.90 & 4.04 \\
\hline $\mathrm{H}$ & Acceso a infraestructura física & 6.72 & 6.06 & 7.44 & 5.76 & 6.17 & 6.38 \\
\hline I & Normas sociales y culturales & 5.35 & 5.78 & 5.05 & 5.01 & 4.85 & 4.76 \\
\hline
\end{tabular}

Figura 5: Factores ecosistema pro emprendimiento

Fuente: (GEM, 2017)

Los factores descritos señalan una escala de 1 a 9 , en este contexto se puede percibir de manera general que tanto la región como las economías de eficiencia sus resultados no son óptimos, por cuanto la mayor parte de factores se encuentran por debajo de la media.

Al analizar el caso ecuatoriano se puede determinar los componentes que afectan directamente al emprendimiento en las pequeñas y medianas empresas: el apoyo financiero es sin duda el de mayor relevancia ya que no se encuentra diseñado para brindar un apalancamiento financiero óptimo, con tasas de interés en dólares que no corresponden a una oferta coherente para su desenvolvimiento; la regulación de políticas gubernamentales mantienen barreras para la creación y consolidación de empresas, marcos jurídicos rígidos en el campo laboral, continuos cambios tributarios entre otros aspectos.

Por otra parte dentro de los aspectos positivos que mantiene Ecuador son la educación formal primaria, secundaria y de nivel superior enfocada a la administración y gestión empresarial superando la media de la región y a las economías de eficiencia; el acceso a infraestructura física desataca la existencia de vialidad, telecomunicaciones así como el acceso a tecnologías de la información a bajo costo; normas sociales y culturales mismas que han tenido un desarrollo por la valoración personal y fortalecimiento de la identidad ecuatoriana como punto referencial de desarrollo. 


\section{Conclusiones}

En los últimos años han existido distintas iniciativas desde diversos ámbitos, con intentos de establecer estrategias nacionales para el emprendimiento sin resultados concretos aún. Se requiere de un conjunto de políticas, reglamentaciones, programas y proyectos en ejecución que lleven al Ecuador hacia un ecosistema que favorezca el emprendimiento de tal manera que permita que éste sea realmente fuente de desarrollo económico. Por ello, y más aún en tiempos de mayor austeridad esta priorización debe ser inmediata.

Como resultado de este análisis se puede acotar que los impedimentos críticos de los emprendimientos ecuatorianos que no generan impacto ni dinamismo son el casi nulo enfoque de internacionalización, acceso a fuentes de financiamiento, innovación de procesos y absorción tecnológica, así como la baja calificación de talento humano en negocios nacientes y nuevos; con la contradicción de ser en la región un referente en educación y formación pro emprendimiento.

Así, las políticas claves serían aquellas orientadas a fomentar la internacionalización a través de un mayor acceso a mercados internacionales, dinamismo en organismos como Pro Ecuador, Ministerio de Comercio Exterior y embajadas; establecer planes de acción conjuntos orientados a las distintas etapas del emprendimiento: capital semilla, capital de riesgo, inversiones ángeles y el fomento de un mercado bursátil (ya desarrollado por los vecinos Perú y Colombia).

El acceso a nuevos mercados a través de acuerdos comerciales de integración obliga a las empresas nacionales, tanto nuevas como establecidas a aumentar sus esfuerzos en innovación a fin de ser capaces de competir con empresas internacionales.

Los componentes citados confluyen una macro orientación hacia el dinamismo del emprendimiento previendo empresas más innovadoras, con capacidad de capitalización y orientadas hacia un mercado exterior. Todos estos esfuerzos deben buscar articular el entorno en función del macro objetivo: conseguir emprendimientos dinámicos establecidos.

Otro aspecto relevante es fomentar de manera conjunta el sector privado y la academia, emprendimientos dispuestos a contribuir significativamente ya no solo a la subsistencia de los propios emprendedores sino también al nivel de desarrollo del país.

En este sentido la construcción del conocimiento enfocado en la innovación y emprendimiento es sin duda el pilar fundamental para la consolidación de talento humano de excelencia, mismo que faculte un creciente indicador EEA; es menester desarrollar mejoras a las mallas curriculares de las instituciones de educación superior donde la fusión del conocimiento académico - teórico y la práctica reflejen los resultados de aprendizaje, a través de la orientación a problemáticas de emprendimientos locales desarrollados en escenarios ecuatorianos actuales, la determinación de factores para el desarrollo de diferenciación de productos y servicios, directrices de internacionalización, determinación de oportunidades de negocio en escenarios locales e internacionales, acceso a información a través de plataformas como The International Trade Center con implementación de laboratorios cien por ciento funcionales donde el docente y el alumno puedan levantar información en tiempo real, medir escenarios y desarrollar emprendimientos. 
Parte fundamental del desarrollo de negocios por oportunidad son los programas orientados al emprendimiento donde se debe fomentar una actitud y cultura positiva hacia la creación de empresas, diseñadas en competencias que conducen al autoempleo, autosuficiencia económica, generación de empleo, enfoque de internacionalización, sostenibilidad, crecimiento de la pequeña empresa contemplando escenarios y factores determinantes desde una perspectiva prospectiva y efectiva.

En síntesis, el emprendedor ecuatoriano debe estar conectado e interrelacionado con su entorno y realidad, debe formarse con una mirada que integre las dimensiones sociales, el contexto temporal y la identificación de oportunidades con enfoque de internacionalización, debe aliarse a las estructuras de fomento para el desarrollo, participar activamente con la academia y los organismos públicos que fomenten el emprendimiento, luchar por espacios a través de asociaciones que busquen mejores prestaciones conjuntas referentes a financiamiento, infraestructura, asesoría comercial, legal, dinámicas de mercado y fuentes de información de calidad, transformando escenarios de apoyo con alianzas estratégicas que permitan aportar conocimiento e innovación; el escenario está planteado es necesario actuar, gestionar y dinamizar el emprendimiento en el Ecuador.

\section{Bibliografía}

Alianza para el Emprendimiento y la Innovación. (2014). AEI. Obtenido de http://aei.ec/emprendedor2/somos/

Araque, W. (2015). Emprendimiento en Ecuador. Ekos Negocio, 72-78.

Coduras, A., Levie, J., \& Kelley, D. (2010). Global Entrepreneurship Monitor Special Report: A Global PErspective on Entrepreneurship Education and Training.

Comisión Europea . (2009). El espíritu empresarial en la educación y formación de profesionales. Informe final del grupo de expertos.

Corporación EKOS media. (2010). Grandes Empresas en Ecuador. Quito: Ediecuatorial.

Covin. (2007). Strategic use of corporate venturing. Entrepreneurship Theory and Practice 31(2), 183207.

Drucker. (1985). Innovation and Entrepreneurship. . New York: Harper y Row.

GEM. (2015). Global Entrepreneurship Monitor Ecuador - 2015.

GEM. (2017). Global Entrepreneurship Monitor Ecuador 2016. ESPAE - ESPOL.

Global Entrepreneurship Monitor. (2017). 2016 / 17 Global Report. Obtenido de http://gem.ufm.edu/que-es-gem/

Grisales, C. P. (2012). Taller \#1 Emprendimiento. Obtenido de https://docs.google.com/document/d/193dA3n2hw2-

K3NR6hcjx9pOzvE9kYy9GNtZcLZu_Dn8/edit 
INEC. (2014). Mirador Obtenido de http://aplicaciones3.ecuadorencifras.gob.ec/VDATOS2war/paginas/administracion/mirador.xhtml

Jaramillo, L. (7 de 12 de 2008). LUMEN. Obtenido de https://guayacan.uninorte.edu.co/divisiones/iese/lumen/ediciones/7/articulos/emprendimiento .pdf

Julien, P. (2005). Emprendimiento regional y economía del conocimiento: una metáfora de las novelas policiacas. Sello Javeriano.

Kelley, D. (2011). Global entrepreneurship monitor 2010. Babson College and Universidad Del Desarrollo.

Lasio, V., Caicedo, G., Ordeñana, X., \& Izquierdo, E. (2014). Global Entrepreneurship Monitor. Obtenido de http://www.gemconsortium.org/report

LOES. (2010). Título VI, Capítulo I.

McClelland. (1961). The achieving society. Princeton. NJ: Van Norstrand Company. Inc.

Michelacci, C. (2003). Low Returns in RyD Due to the Lack of Entrepreneurial Skills. The Economic Journal, 113(484),, 207-225.

Nodal. (08 de Enero de 2016).

Oosterbeek, H., \& Van Praag, M. (2010). The impact of entrepreneurship education on entrepreneurship skills and motivation. European Economic Review, 442-454.

Osorio, F., Gálvez, E., \& Murillo, G. (2010). La estrategia y el emprendedor: diversas perspectivas para el análisis. Cuadernos de Administración Universidad del Valle, 43, 87-107.

Real Academia Española. (1732). Diccionario de Autoridades - Tomo III.

Say. (1821). Tratado de Economia Politica: ó exposición sencilla del modo con. (Vol. 2). Imp. de Fermin Villalpando.

Schumpeter. (1911). The theory of economic development . Harvard economic studies.

Shane, S. (2002). University technology transfer to entrepreneurial companies. Journal of Business Venturing, $\mathrm{n}^{\circ} .17,537-552$.

Simón, E. K. (2003). La creación de empresas de base tecnológica: Una experiencia práctica. Proyecto para la promoción de empresas innovadoras de base tecnológica. España.

Solow, R. M. (1957). Technical change and the aggregate production function. The review of Economics and Statistics, 312-320. 